\title{
10 MODELO DE INTERVENÇÃO PSICOTERAPÊUTICA EM ENFERMAGEM: PRINCÍPIOS ORIENTADORES PARA A IMPLEMENTAÇÃO NA PRÁTICA CLÍNICA
}

\author{
| Francisco Sampaio' ${ }^{1}$ Carlos Sequeira ${ }^{2}$; Teresa Lluch Canut ${ }^{3} \mid$
}

\section{RESUMO}

CONTEXTO: Apesar de, em Portugal, se encontrar regulamentada a competência dos enfermeiros especialistas em Enfermagem de Saúde Mental para realizar intervenções psicoterapêuticas, não existia até agora qualquer modelo de intervenção psicoterapêutica em Enfermagem que permitisse sistematizar essa prática. Tendo este sido desenvolvido e avaliado quanto à sua eficácia, importa agora que a sua utilização seja clara para que este possa ser transposto para a prática clínica.

OBJETIVO: Descrever os passos necessários para a operacionalização do modelo de intervenção psicoterapêutica em Enfermagem nos contextos da prática clínica.

MÉTODOS: Este trata-se de um artigo de boas práticas no qual são apresentadas as estratégias a adotar para a operacionalização do modelo de intervenção psicoterapêutica em Enfermagem na prática clínica, bem como algumas dificuldades relacionadas com a sua utilização.

RESULTADOS: Numa sessão 0 o utente deve ser avaliado no sentido de apreciar se este cumpre os critérios de inclusão e não apresenta qualquer critério de exclusão para ser intervencionado com recurso ao modelo de intervenção psicoterapêutica em Enfermagem. Após a identificação do(s) diagnóstico(s) de Enfermagem prioritário(s) (CIPE ou NANDA-I) importa compreender a origem/causa do(s) problema(s) e, de acordo com a mesma, selecionar a(s) intervenção/ões psicoterapêutica(s) de Enfermagem (NIC) a utilizar (mediante o preconizado na obra "Ligações NANDANOC-NIC: Condições clínicas: Suporte ao raciocínio e assistência de qualidade").

CONCLUSÕES: O modelo de intervenção psicoterapêutica em Enfermagem parece ser uma mais-valia pela possibilidade de sistematizar a prática dos enfermeiros especialistas em Enfermagem de Saúde Mental. Contudo, é ainda necessário criar ferramentas que permitam simplificar a sua transposição para a prática clínica.

\section{PALAVRAS-CHAVE: Enfermagem; Enfermagem psiquiátrica; Prática profissional; Psicoterapia}

\section{RESUMEN}

"Modelo de intervención psicoterapéutica en enfermería: Principios orientadores para la implementación en la práctica clínica"

CONTEXTO: Aún que, en Portugal, esté regulada la competencia de los enfermeros especialistas en Enfermería de Salud Mental para realizar intervenciones psicoterapéuticas, no había hasta ahora ningún modelo de intervención psicoterapéutica en Enfermería que permitiría sistematizar esta práctica. Al haber sido desarrollado y evaluado en cuanto a su eficacia, es importante que su utilización sea clara para que éste pueda transponerse a la práctica clínica. OBJETIVO: Describir los pasos necesarios para la operacionalización del modelo de intervención psicoterapéutica en Enfermería en los contextos de la práctica clínica.

MÉTODOS: Este se trata de un artículo de buenas prácticas en el cual se presentan las estrategias a adoptar para la operacionalización del modelo de intervención psicoterapéutica en Enfermería en la práctica clínica, así como algunas dificultades relacionadas con su utilización.

RESULTADOS: En una sesión 0 el paciente debe ser evaluado en el sentido de apreciar si éste cumple los criterios de inclusión y no presenta ningún criterio de exclusión para ser intervenido con recurso al modelo de intervención psicoterapéutica en Enfermería. Después de la identificación del/de los diagnóstico(s) de Enfermería prioritario(s) (CIPE o NANDA-I), es importante comprender el origen/causa del/de los problema(s) y, de acuerdo con la misma, seleccionar la(s) intervención(es) psicoterapéutica(s) de Enfermería (NIC) a utilizar (mediante el preconizado en la obra "Vínculos de NOC y NIC a NANDA-I: Soporte para el razonamiento crítico y la calidad de los cuidados") CONCLUSIONES: El modelo de intervención psicoterapéutica en Enfermería parece ser una plusvalía por la posibilidad de sistematizar la práctica de los enfermeros especialistas en Enfermería de Salud Mental. Sin embargo, aún es necesario crear herramientas que permitan simplificar su transposición a la práctica clínica.

\section{DESCRIPTORES: Enfermería; Enfermería psiquiátrica; Prác- tica profesional; Psicoterapia}

\begin{abstract}
"Psychotherapeutic intervention model in nursing: Guiding principles for implementation in clinical practice"

BACKGROUND: Although in Portugal the competence of psychiatric nurses to perform psychotherapeutic interventions is regulated, there was no psychotherapeutic intervention model in nursing available to systematize this practice. Having a model been developed and evaluated for its efficacy, it is now important to make its use clear in order to transpose it into clinical practice. AIM: To describe the necessary steps for the operationalization of the psychotherapeutic intervention model in nursing in the clinical practice contexts.

METHODS: This is an article of best practices in which the strategies to be adopted for the operationalization of the psychotherapeutic intervention model in nursing into clinical practice are presented, as well as some difficulties related to its use.

RESULTS: In a session 0 the patient should be evaluated in order to assess whether he/she meets the inclusion criteria and does not present any exclusion criterion to be intervened using the psychotherapeutic intervention model in nursing. After identifying the priority nursing diagnosis/es (ICNP or NANDA-I) it is important to understand the origin/cause of the problem(s) and, according to it, to select the psychotherapeutic intervention(s) (according to what is recommended in the book "NOC and NIC linkages to NANDA-I and clinical conditions: Supporting critical reasoning and quality care").

CONCLUSIONS: The psychotherapeutic intervention model in nursing seems to be an added value for the possibility of systematizing the practice of the psychiatric nurses. However, it is still necessary to develop tools in order to simplify its transposition into clinical practice.
\end{abstract}

\section{KEYWORDS: Nursing; Psychiatric nursing; Professional prac- tice; Psychotherapy}

Submetido em 28-03-2018

Aceite em 14-04-2018

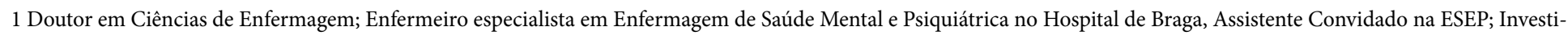
gador Integrado no CINTESIS , grupo de investigação “NursID - Inovação e Desenvolvimento em Enfermagem”; Francisco.Sampaio@hospitaldebraga.pt

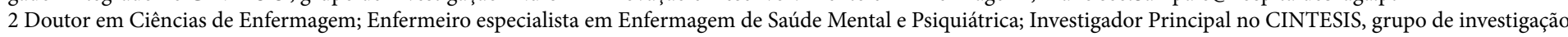
"NursID - Inovação e Desenvolvimento em Enfermagem”; Professor Coordenador na Escola Superior de Enfermagem do Porto, Portugal, carlossequeira@esenf.pt

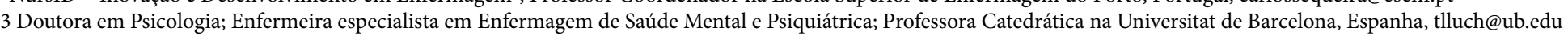




\section{CONTEXTO}

Ao longo dos tempos recentes tem vindo a considerarse, crescentemente, que aquilo que distingue, na prática clínica (execução de cuidados), um enfermeiro de cuidados gerais de um enfermeiro especialista em Enfermagem de Saúde Mental e Psiquiátrica (ESMP) é, sobretudo, a competência específica que este último tem para prestar cuidados de âmbito psicoterapêutico. Esta trata-se de uma competência assumida em Diário da República e expressa no Regulamento n. ${ }^{\circ}$ 129/2011. Apesar da expressão regulamentar da competência supramencionada os enfermeiros especialistas em ESMP não dispunham, de acordo com a pesquisa bibliográfica realizada, de qualquer modelo de intervenção psicoterapêutica em Enfermagem que permitisse guiar e sistematizar as suas práticas. Tendo por base essa lacuna que, crê-se, dificulta uma consistente transposição da intervenção psicoterapêutica para a prática clínica por parte dos enfermeiros especialistas em ESMP, foi criado um modelo de intervenção psicoterapêutica em Enfermagem baseado, mormente, em teorias de Enfermagem, nos princípios basilares do processo de Enfermagem e em taxonomias de Enfermagem (Sampaio, Sequeira, \& Lluch Canut, 2017a). O modelo em causa foi avaliado, no que diz respeito à sua eficácia a curto prazo, junto de utentes psiquiátricos adultos com o diagnóstico de Enfermagem "Ansiedade", tendo apresentado resultados positivos, estatisticamente significativos, tanto no que concerne ao nível de ansiedade como ao autocontrolo da ansiedade (inclusivamente por comparação com o grupo de controlo, submetido exclusivamente a terapêutica psicofarmacológica) (Sampaio, Araújo, Sequeira, Lluch Canut, \& Martins, 2018).

Ainda que o desenvolvimento de um modelo de intervenção psicoterapêutica em Enfermagem, cuja estrutura parece adequada face aos resultados decorrentes do estudo de avaliação da sua eficácia, seja encarado como um passo importante para a sistematização das práticas psicoterapêuticas dos enfermeiros especialistas em ESMP, torna-se essencial que estes profissionais se familiarizem com o mesmo, isto é, compreendam claramente os pressupostos inerentes à sua utilização na prática clínica. Previamente à apresentação do modelo e da sua operacionalização em contextos da prática clínica importa referir que este se trata de um modelo de intervenção psicoterapêutica de Enfermagem e não de Psicoterapia. Na distinção dos conceitos ressalvase que a Psicoterapia envolve um contacto sistemático e limitado no tempo entre uma pessoa em distress e alguém que procura reduzir esse distress por via da produção de mudanças nos sentimentos, atitudes e comportamento com recurso a uma relação terapêutica (Frank \& Frank, 1993; Frank in Bloch, 2006). Por outro lado, a intervenção psicoterapêutica de Enfermagem consiste na aplicação informada e intencional de técnicas de Psicoterapia e assenta nos seguintes princípios: a) é uma intervenção estruturada, sistematizada, progressiva e sequencial; b) é realizada por um enfermeiro especialista em ESMP ou por um enfermeiro de cuidados gerais com formação específica e competências certificadas pela Ordem dos Enfermeiros; c) é uma intervenção baseada em um ou mais racionais teóricos de Psicoterapia; d) é uma intervenção decorrente da identificação de um diagnóstico de Enfermagem no âmbito da Saúde Mental; e) é uma intervenção na qual o utente apresenta um papel ativo e tem, necessariamente, alguma consciência de si; f) é uma intervenção com um número de sessões entre três e doze; g) é uma intervenção que tem, na sua base, a relação terapêutica e a comunicação interpessoal entre o enfermeiro e o utente; h) é uma intervenção cujo objetivo é a mudança ou a redução de um estado percebido como negativo pelo utente através da definição prévia de metas mútuas entre o enfermeiro e o utente (Sampaio, Sequeira, \& Lluch Canut, 2014). De forma sumária, a intervenção psicoterapêutica de Enfermagem distingue-se da Psicoterapia "tradicional" pelo facto de ser mais limitada no tempo, ter como ponto de partida um diagnóstico de Enfermagem, poder decorrer noutros settings que não, exclusivamente, o de gabinete / consultório e por se circunscrever à utilização de técnicas de Psicoterapia sob a forma de intervenções integradas na Classificação das Intervenções de Enfermagem (NIC) (por exemplo, reestruturação cognitiva [4700] ou treino de controlo de impulsos [4370]) (Bulechek, Butcher, Dochterman, \& Wagner, 2012).

\section{OBJETIVO}

Com o presente artigo pretende-se, acima de tudo, apresentar os dados necessários para a operacionalização do modelo de intervenção psicoterapêutica em Enfermagem desenvolvido. Desse modo, como fim último, pretende-se que os enfermeiros especialistas em ESMP tenham acesso a toda a informação entendida como necessária para a aplicação do modelo nos contextos da prática clínica visando a sua transposição para os mesmos. 


\section{MÉTODOS}

Este trata-se de um artigo de boas práticas no qual se apresenta o modelo de intervenção psicoterapêutica em Enfermagem desenvolvido. Para a criação do modelo em causa foram realizados, inicialmente, uma revisão narrativa da literatura (visando identificar as comunalidades relativas à intervenção psicoterapêutica conduzida por enfermeiros) (Sampaio, Sequeira, \& Lluch Canut, 2015) e um estudo de focus groups (visando perceber as caraterísticas que, de acordo com um grupo de peritos composto por enfermeiros portugueses e espanhóis, deveria apresentar um modelo de intervenção psicoterapêutica em Enfermagem) (Sampaio, Sequeira, \& Lluch Canut, 2017b). Tendo por base o cruzamento dos dados obtidos nos dois estudos iniciais foi criado um esboço do modelo tendo este sido submetido a um grupo de peritos, previamente selecionados com recurso a critérios de inclusão pré-definidos, por via de um estudo e-Delphi modificado para validação do conteúdo do mesmo (Sampaio, Sequeira, \& Lluch Canut, 2017a).

Para além da estrutura do modelo de intervenção psicoterapêutica em Enfermagem desenvolvido, neste artigo de boas práticas apresentar-se-ão alguns princípios orientadores para a utilização do mesmo nos contextos da prática clínica. Assim, este trata-se de um artigo que tem também uma finalidade informativa / formativa que deverá servir para potenciar a translação do conhecimento produzido para a prestação de cuidados, com potencial benefício para os utentes.

\section{RESULTADOS E DISCUSSÃO}

O modelo de intervenção psicoterapêutica em Enfermagem trata-se, essencialmente, de um modelo estrutural ("chapéu de chuva") ao qual podem ser alocadas todas as intervenções psicoterapêuticas realizadas por enfermeiros especialistas em ESMP. Destaca-se no modelo, para além da sua estrutura-base, o facto de este assentar integralmente em taxonomias de Enfermagem, incluindo a da NANDA International, Inc. (NANDA-I), a Classificação Internacional para a Prática de Enfermagem (CIPE), a NIC e a Classificação dos Resultados de Enfermagem (NOC). O recurso a diversas taxonomias relaciona-se com o aumento da potencialidade de se utilizar o modelo em localizações geográficas diversas (Fig. 1).

Figura 1 - Modelo de intervenção psicoterapêutica em enfermagem.

\begin{tabular}{|c|c|c|c|}
\hline 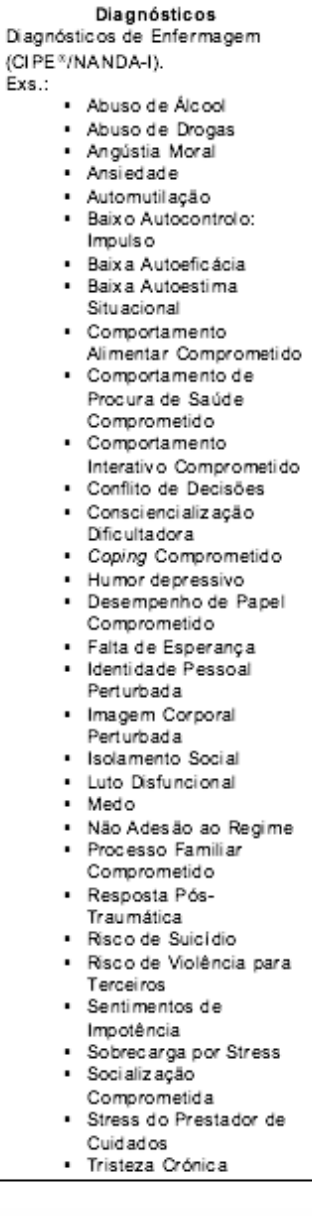 & 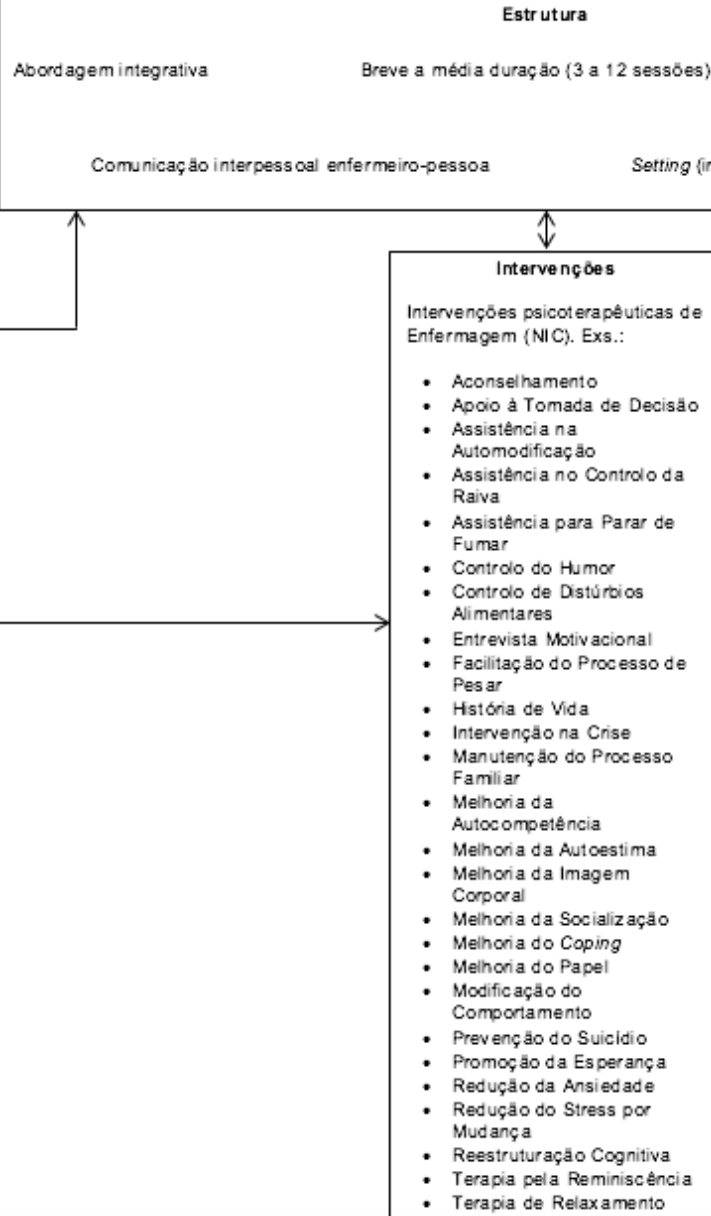 & $\begin{array}{l}\qquad \text { Relaç ào ter apétutic a } \\
\text { ternamento ou ambulatónio) } \\
\text { T }\end{array}$ & 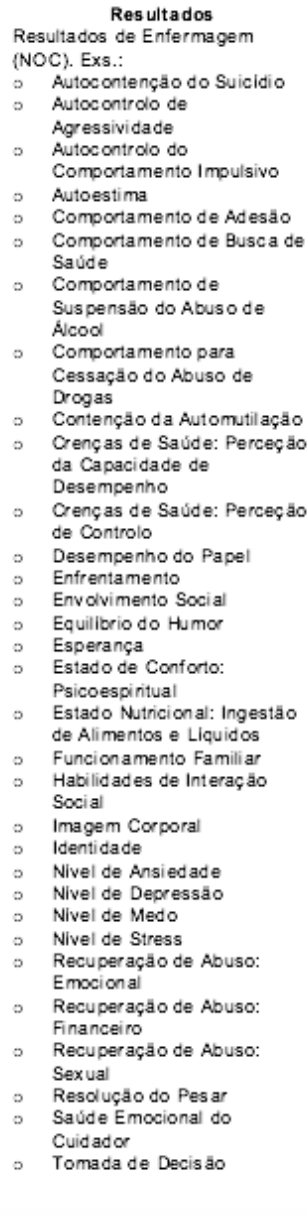 \\
\hline
\end{tabular}


Antes de se avançar para a execução de intervenção psicoterapêutica integrada no modelo importa, numa sessão 0 , realizar uma avaliação mental abrangente do utente. Assim, nessa avaliação devem ser tidos em consideração os aspetos apresentados na Tabela 1 visando, igualmente, apreciar se o utente pode ou não ser incluído na intervenção (por via da avaliação da presença de um ou mais dos critérios de exclusão) (Sampaio, 2017).

Tabela 1 - Domínios da Avaliação Inicial e Critérios de Exclusão de Utentes

\begin{tabular}{|l|l|}
\hline \multicolumn{2}{|c|}{ Avaliação Inicial (Domínios a Considerar) } \\
\hline Comportamento & Preservação do status mental \\
\hline Processos de pensamento & Linguagem \\
\hline Estado afetivo & Dificuldades de perceção \\
\hline Memória & $\begin{array}{l}\text { Status físico e satisfação das } \\
\text { necessidades }\end{array}$ \\
\hline Capacidade de adaptação & Capacidade funcional \\
\hline Aparência física & $\begin{array}{l}\text { Necessidade de conhecimen- } \\
\text { tos em saúde }\end{array}$ \\
\hline $\begin{array}{l}\text { Risco de agressão auto ou } \\
\text { heterodirigida }\end{array}$ & Critérios de Exclusão de Utentes \\
\hline \multicolumn{2}{|c|}{} \\
\hline Estado confusional & Défice cognitivo grave \\
\hline Agitação psicomotora & $\begin{array}{l}\text { Sintomatologia heteróloga } \\
\text { grave }\end{array}$ \\
\hline
\end{tabular}

Tratando-se este de um modelo que assenta numa abordagem integrativa é realizada uma combinação de diferentes técnicas (intervenções), oriundas de diversas escolas de Psicoterapia, que visa ir de encontro às reais necessidades do utente (Norcross in Norcross \& Goldfried, 2005). Essa abordagem, patente na Fig. 2, implica necessariamente que o enfermeiro especialista em ESMP, ao selecionar a "linha" psicoterapêutica a utilizar, faça uso dos princípios inerentes à mesma. Assim, por exemplo, caso o enfermeiro considere que o utente tem capacidade intrínseca para a resolução de determinado problema e, como tal, opte por uma intervenção psicoterapêutica da linha humanista (ex.: aconselhamento), é essencial que este mantenha uma postura assente na congruência, na empatia e na aceitação incondicional, que encare o utente tendo em conta a sua natureza individual e não segundo o seu contexto patológico, e que o ajude na concretização da sua tendência autoatualizante.

Importa, ainda assim, distinguir claramente a abordagem integrativa preconizada pelo modelo de intervenção psicoterapêutica em Enfermagem do ecletismo técnico. Assim, enquanto neste último se defende uma combinação paradigmática de técnicas com base na eficácia clínica observada ou presumida (Safran \& Messer, 1998), sem que seja estritamente necessário adotar o sistema teórico associado a estas, a abordagem integrativa envolve, igualmente, a ligação entre a teoria, a evidência e a técnica, isto é, foca-se na relação entre a prática e as suas bases teóricas e empíricas (Norcross in Norcross \& Goldfried, 2005).

Figura 2 - Abordagem integrativa do modelo de intervenção psicoterapêutica em enfermagem

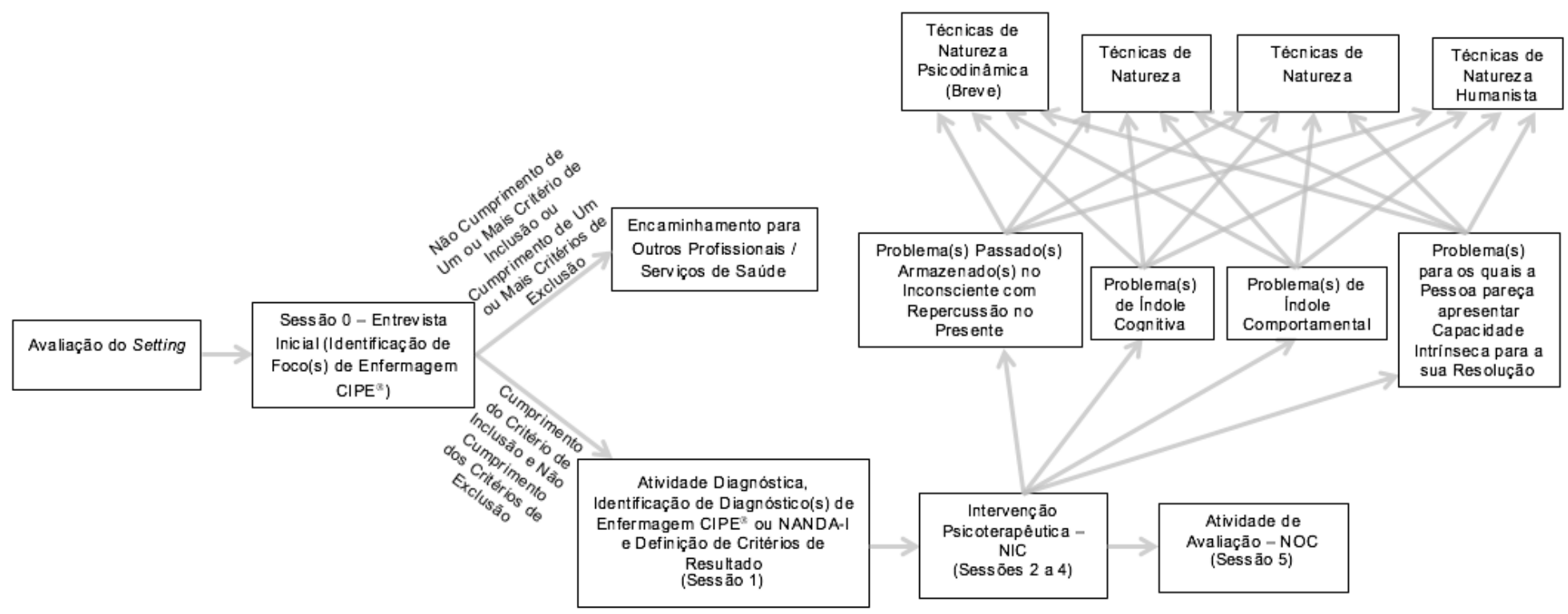


Porquanto, é essencial que, ao fazer uso do modelo desenvolvido, o enfermeiro especialista em ESMP se baseie em alguns princípios teóricos já existentes, não apenas no que concerne à postura a adotar mediante a(s) intervenção/ões selecionada(s) mas também ao nível das intervenções passíveis de ser selecionadas para dar resposta ao(s) diagnóstico(s) de Enfermagem identificado(s). Para a realização deste trabalho é fulcral o recurso ao livro "Ligações NANDA-NOC-NIC: Condições clínicas: Suporte ao raciocínio e assistência de qualidade" (Johnson et al., 2013). Assim, o exercício a realizar deve passar por procurar o diagnóstico de Enfermagem identificado (ex.: "Baixa autoestima situacional") e qual o resultado que se pretende alcançar (ex.: promover a autoestima, traduzido pelo resultado NOC "Autoestima"). De entre as intervenções (principais, sugeridas e opcionais) alocadas ao diagnóstico de Enfermagem "Baixa autoestima situacional" - resultado "Autoestima", importa selecionar unicamente aquelas que se tratam de intervenções psicoterapêuticas de Enfermagem (ver Fig. 1, coluna “Intervenções”) (Tabela 2).

Tabela 2 - Ligações entre o Diagnóstico de Enfermagem

"Baixa Autoestima Situacional", as Intervenções

Psicoterapêuticas NIC e os Resultados NOC

\begin{tabular}{|l|l|l|}
\hline $\begin{array}{l}\text { Diagnóstico de } \\
\text { Enfermagem } \\
\text { (código CIPE/ } \\
\text { NANDA-I) }\end{array}$ & $\begin{array}{l}\text { Intervenções de Enfer- } \\
\text { magem } \\
\text { (código NIC) }\end{array}$ & $\begin{array}{l}\text { Resultados de } \\
\text { Enfermagem } \\
\text { (código } \\
\text { NOC) }\end{array}$ \\
\hline $\begin{array}{l}\text { Baixa autoestima } \\
\text { situacional } \\
(10000844 / 00120)\end{array}$ & $\begin{array}{l}\text { Intervenção Principal } \\
\text { Melhoria do coping (5230) } \\
\text { Melhoria da autoestima } \\
\text { (5400) } \\
\text { Intervenções Sugeridas } \\
\text { Treino da assertividade } \\
(4340)\end{array}$ & $\begin{array}{l}\text { Autoestima } \\
\text { Reestruturação cognitiva } \\
(4700)\end{array}$ \\
& $\begin{array}{l}\text { Aconselhamento (5240) } \\
\text { Intervenção na crise } \\
(6160)\end{array}$ \\
& $\begin{array}{l}\text { Facilitação do processo de } \\
\text { pesar (5290) } \\
\text { Intervenções Opcionais } \\
\text { Melhoria da socialização } \\
\text { (5100) } \\
\text { História de vida }\end{array}$ & \\
\hline
\end{tabular}

No exemplo apresentado o enfermeiro especialista em ESMP, necessariamente (por se tratar de uma intervenção principal), deve realizar as intervenções psicoterapêuticas NIC "Melhoria do coping" e "Melhoria da autoestima”. Adicionalmente pode realizar uma ou mais das intervenções sugeridas e/ou opcionais mas privilegiando, naturalmente, as primeiras.
A intervenção "História de vida" não se encontra classificada na NIC mas, pelo facto de esta classificação não conter quaisquer intervenções do domínio psicodinâmico / psicanalítico, considerou-se relevante a sua inclusão no modelo de intervenção psicoterapêutica em Enfermagem. Pelo facto de, claramente, a autoestima poder ser tratada por via da abordagem psicodinâmica (ex.: Ritter, Leichsenring, Strauss, \& Stangier, 2013), optou-se também por incluir a intervenção como "opcional” para o diagnóstico de Enfermagem "Baixa autoestima situacional", devendo recorrer-se à mesma no caso de o enfermeiro considerar que a baixa autoestima apresentada pelo utente tem relação com life events passados.

É evidente neste processo que, pese embora a lógica metodológica, a sua operacionalidade é limitada pela necessidade de consulta e análise de um livro que, mesmo que presente nos contextos da prática clínica, torna o processo algo moroso e pouco intuitivo. Este é, claramente, um dos aspetos a melhorar para que se cumpra a pretensão de ver o modelo de intervenção psicoterapêutica em Enfermagem implementado nos contextos da prática clínica. Assim, parece necessário desenvolver um documento (manual de suporte) no qual, com maior rapidez e facilidade, os enfermeiros especialistas em ESMP consigam identificar as intervenções psicoterapêuticas NIC passíveis de ser realizadas para dar resposta ao(s) diagnóstico(s) de Enfermagem nomeado(s), bem como os resultados NOC que podem ser utilizados para proceder à avaliação da eficácia das intervenções. Outra hipótese, mais inovadora, passaria por criar uma aplicação (app), utilizável com recurso a um computador, tablet ou smartphone, que tornasse acessível aos enfermeiros, de forma intuitiva, a informação supramencionada (eventualmente incluindo um sistema de apoio à decisão que os auxiliasse a decidir qual/quais a(s) intervenção/ões mais adequada(s) face ao(s) problema(s) apresentado(s) pelo utente).

Adicionalmente, e pese embora o Regulamento n. ${ }^{\circ}$ $129 / 2011$ afirme que os enfermeiros especialistas em ESMP têm a competência específica para prestar cuidados de âmbito psicoterapêutico, fica a ideia da necessidade de formação adicional dos mesmos neste domínio. Assim, crê-se que a criação de uma Pós-Graduação em Intervenção Psicoterapêutica em Enfermagem poderia ser útil para: a) clarificar e treinar as atividades que concretizam cada intervenção psicoterapêutica NIC; b) clarificar e treinar a operacionalização do modelo de intervenção psicoterapêutica em Enfermagem. 
$\mathrm{Na}$ prática clínica, e aquando da implementação do modelo, a principal dificuldade identificada foi, indubitavelmente, o baixo nível de literacia em saúde dos utentes. É sabido que Portugal, em comparação com os oito países participantes no estudo Health Literacy Survey (Áustria, Alemanha, Bulgária, Espanha, Grécia, Irlanda, Holanda e Polónia), apresentou valores ligeiramente mais baixos no índice geral de literacia em saúde do que a média desses países (Pedro, Amaral, e Escoval, 2016). No contexto psiquiátrico, sendo frequente o contacto com utentes que se apresentam muito vulneráveis no campo da literacia em saúde (pelo baixo nível de escolaridade, rendimentos inferiores a 500€, doença[s] prolongada[s] e/ou "má" autoperceção de saúde) (Espanha, Ávila, e Mendes, 2016), a situação parece ainda mais problemática. Assim, sendo evidente a tendencial crença numa abordagem essencialmente psicofarmacoterapêutica, não raras vezes os utentes apresentaram alguma desconfiança relativamente à (adicional) abordagem psicoterapêutica proposta embora, paradoxalmente, a taxa de dropouts do grupo experimental (31\%) tenha estado em linha com a apresentada na literatura internacional para a intervenção psicoterapêutica limitada no tempo (Sledge, Moras, Hartley, \& Levine, 1990).

Adicionalmente, foram sentidas dificuldades relacionadas com a disponibilidade dos utentes para a intervenção. Assim, e sobretudo para os ativos empregados, a marcação de sessões semanais, não raras vezes, implicava com o horário laboral dos utentes. Como tal, houve a necessidade de concentrar muitas sessões de intervenção no final da tarde e, ocasionalmente, até mesmo em horário pós-laboral (impraticável num contexto [real] de Consulta Externa dado o horário de encerramento da mesma).

Finalmente, o facto de os utentes apresentarem, com alguma frequência, um discurso e/ou comportamento sugestivo de um Quociente de Inteligência (QI) relativamente baixo, foi também dificultador do trabalho inerente à intervenção psicoterapêutica. Estudos (ex.: Mortensen, Sørensen, Jensen, Reinisch, \& Mednick, 2005) indicam que o score médio obtido pelas pessoas com doença mental em testes de QI é relativamente baixo e que estes resultados não refletem, necessariamente, efeitos da doença mental, mas antes valores que vão de encontro ao QI pré-mórbido. Assim, em muitas sessões, dada a dificuldade de se realizar trabalho psicoterapêutico propriamente dito, entenda-se, visando a mudança de cognições, comportamentos e/ou emoções, fez-se uso da escuta ativa enquanto técnica de comunicação predominante para a construção e manutenção da relação terapêutica, tendo sido privilegiada a comunicação expressiva por parte do utente em detrimento do incentivo ao raciocínio orientado para a mudança.

\section{CONCLUSÕES}

Ao longo dos últimos cinco anos tem vindo a ser realizada investigação no sentido de desenvolver e avaliar um modelo de intervenção psicoterapêutica em Enfermagem. Tendo-se chegado à sua versão final e avaliado a sua eficácia a curto prazo, em contexto de ambulatório, em utentes psiquiátricos adultos que apresentavam o diagnóstico de Enfermagem "Ansiedade", tanto no que concerne ao nível de ansiedade como ao autocontrolo da mesma, importava apresentar alguns princípios orientadores que permitissem aos enfermeiros especialistas em ESMP transpô-lo para os contextos da prática clínica.

O presente artigo visou servir esse desígnio, descrevendo os passos a seguir para a implementação do modelo de intervenção psicoterapêutica em Enfermagem. Ficam evidentes algumas limitações ainda existentes para que se cumpra esse objetivo mas, acima de tudo, importa destacar os aspetos a atender na investigação futura para que o modelo desenvolvido possa tornar-se cada vez mais comprovado cientificamente e funcional. Assim, para fins de investigação futura, os aspetos seguidamente enumerados afiguram-se como extremamente relevantes:

a) realizar estudos clínicos controlados randomizados que visem avaliar a eficácia do modelo para outros diagnósticos de Enfermagem, não apenas a curto mas também a longo prazo (exemplo: follow-up três e/ou seis meses após o término da intervenção);

b) realizar estudos de custo-efetividade que permitem avaliar se, para além dos benefícios sociais, existem também benefícios económicos, para o Serviço Nacional de Saúde, decorrentes da implementação do modelo nos contextos da prática clínica.

Adicionalmente, como forma de tornar a intervenção ainda mais sistematizada, coloca-se a hipótese de desenvolver programas de intervenção, recorrendo a intervenções psicoterapêuticas NIC, para dar resposta a cada diagnóstico de Enfermagem incluído no modelo. Porém, e ainda que pareça importante testar esta ideia em contexto de investigação, a mesma apresenta dois riscos: 
a) tornar a intervenção psicoterapêutica de Enfermagem estandardizada, ao invés de apenas sistematizada, colocando em causa a individualização na prestação de cuidados;

b) reduzir o envolvimento do enfermeiro na intervenção em decurso da limitação do seu papel no processo de tomada de decisão.

\section{IMPLICAÇÕES PARA A PRÁTICA CLÍNICA}

O presente artigo e, mais concretamente, o modelo de intervenção psicoterapêutica em Enfermagem desenvolvido apresenta implicações para a Enfermagem a três níveis: a) prática clínica; b) ensino; c) investigação. No que diz respeito à prática clínica existe agora um modelo que permite aos enfermeiros especialistas em ESMP guiar e sistematizar a intervenção psicoterapêutica por si conduzida, modelo esse que apresentou resultados positivos no primeiro estudo realizado para avaliação da sua eficácia (a curto prazo) e que, como tal, parece traduzir-se numa mais-valia para os utentes. Caso os estudos de custo-efetividade venham a comprovar, também a esse nível, uma relação favorável, então sob o ponto de vista político o modelo desenvolvido pode traduzir-se numa alternativa viável para o Serviço Nacional de Saúde, que permita ajudar a garantir a sustentabilidade económica do mesmo.

No que concerne ao ensino de Enfermagem as implicações parecem ganhar particular relevo, sobretudo, ao nível do Curso de Pós-Licenciatura de Especialização. Sendo a intervenção psicoterapêutica uma competência específica do enfermeiro especialista em ESMP, a introdução da sua lecionação na formação pós-graduada poderá vir a constituir uma mais-valia num contexto formativo no qual, frequentemente, os profissionais referem a necessidade da existência de modelos que sirvam como guidelines para a sua prática.

Finalmente, no que diz respeito à investigação o modelo de intervenção psicoterapêutica em Enfermagem deixa em aberto uma linha de investigação neste domínio. Tendo sido realizado unicamente um estudo de avaliação da eficácia (a curto prazo) do modelo, para apenas um diagnóstico de Enfermagem, fica em aberto a necessidade de realização de estudos para avaliação da eficácia a curto e longo prazo da eficácia do modelo para todos os outros diagnósticos de Enfermagem integrados no mesmo, bem como de estudos de custoefetividade.

\section{REFERÊNCIAS BIBLIOGRÁFICAS}

Bulechek, G. M., Butcher, H. K., Dochterman, J. M., \& Wagner, C. (2012). Nursing Interventions Classification (NIC) (6 ed.). St. Louis: Elsevier.

Espanha, R., Ávila, P., e Mendes, R. V. (2016). Literacia em saúde em Portugal: Relatório Síntese. Lisboa: Fundação Calouste Gulbenkian.

Frank, J. D. (2006). What is psychotherapy? In S. Bloch (ed.), An introduction to the psychotherapies (pp. 5978). Nova Iorque: Oxford University Press.

Frank, J. D., \& Frank, J. B. (1993). Persuasion and healing: A comparative study of psychotherapy ( $3^{a}$ ed.). Baltimore: John Hopkins University Press.

Johnson, M., Moorhead, S., Bulechek, G., Butcher, H., Maas, M., \& Swanson, E. (2013). Ligações NANDANOC-NIC: Condições clínicas: Suporte ao raciocínio e assistência de qualidade ( $3^{\text {a }}$ ed.). Rio de Janeiro: Elsevier.

Mortensen, E. L., Sørensen, H. J., Jensen, H. H., Reinisch, J. M., \& Mednick, S. A. (2005). IQ and mental disorder in young men. British Journal of Psychiatry, 187(5), 407-415. doi: 10.1192/bjp.187.5.407

Norcross, J. C. (2005). A primer in psychotherapy integration. In J. C. Norcross, \& M. R. Goldfried (eds.), Handbook of psychotherapy integration (pp. 3-23). Nova Iorque: Oxford University Press.

Pedro, A. R., Amaral, O., e Escoval, A. (2016). Literacia em saúde, dos dados à ação: Tradução, validação e aplicação do European Health Literacy Survey em Portugal: Revista Portuguesa de Saúde Pública, 34(3), 259275. doi: 10.1016/j.rpsp.2016.07.002

Regulamento n. ${ }^{\circ}$ 129/2011 (2011). D.R. 2a Série. N. ${ }^{\circ} 35$, 8669-8673.

Ritter, V., Leichsenring, F., Strauss, B. M., \& Stangier, U. (2013). Changes in implicit and explicit self-esteem following cognitive and psychodynamic therapy in social anxiety disorder. Psychotherapy Research, 23(5), 547558. doi: 10.1080/10503307.2013.802824 
Safran, J. D., Abreu, I., Ogilvie, J., \& DeMaria, A. (2011). Does psychotherapy research influence the clinical practice of researcher-clinicians?. Clinical Psychology: Science and Practice, 18(4), 357-371. doi: 10.1111/j.14682850.2011.01267.x

Sampaio, F. (2017). Development and evaluation of a psychotherapeutic intervention model in nursing (Tese de Doutoramento). Instituto de Ciências Biomédical Abel Salazar da Universidade do Porto, Portugal.

Sampaio, F., Araújo, O., Sequeira, C., Lluch Canut, T., \& Martins, T. (2018). A randomized controlled trial of a nursing psychotherapeutic intervention for anxiety in adult psychiatric outpatients. Journal of Advanced Nursing. doi: 10.1111/jan.13520

Sampaio, F., Sequeira, C., \& Lluch Canut, T. (2014). Intervenciones NIC del dominio conductual: Um estúdio de focus group de intervenciones psicoterapêuticas de enfermería. In Asociación Española de Nomenclatura, Taxonomia y Diagnósticos de Enfermería (AENTDE) (ed.), X Simposium AENTDE "Lenguaje enfermero: identidade, utilidad y calidad" (pp. 718-721). Sevilha: AENTDE.
Sampaio, F., Sequeira, C., \& Lluch Canut, T. (2015). Nursing psychotherapeutic interventions: A review of clinical studies. Journal of Clinical Nursing, 24(15-16), 2096-2105. doi: 10.1111/jocn.12808

Sampaio, F., Sequeira, C., \& Lluch Canut, T. (2017a). Content validity of a psychotherapeutic intervention model in nursing: A modified e-Delphi study. Archives of Psychiatric Nursing, 31(2), 147-156. doi: 10.1016/j. apnu.2016.09.007

Sampaio, F., Sequeira, C., \& Lluch Canut, T. (2017b). Contributes for the development of a psychotherapeutic intervention model in nursing: A focus group study in Portugal and Spain. Perspectives in Psychiatric Care. doi: $10.1111 /$ ppc. 12211

Sledge, W. H., Moras, K., Hartley, D., \& Levine, M. (1990). Effect of time-limited psychotherapy on patient dropout rates. American Journal of Psychiatry, 147(10), 1341-1347. doi: 10.1176/ajp.147.10.1341

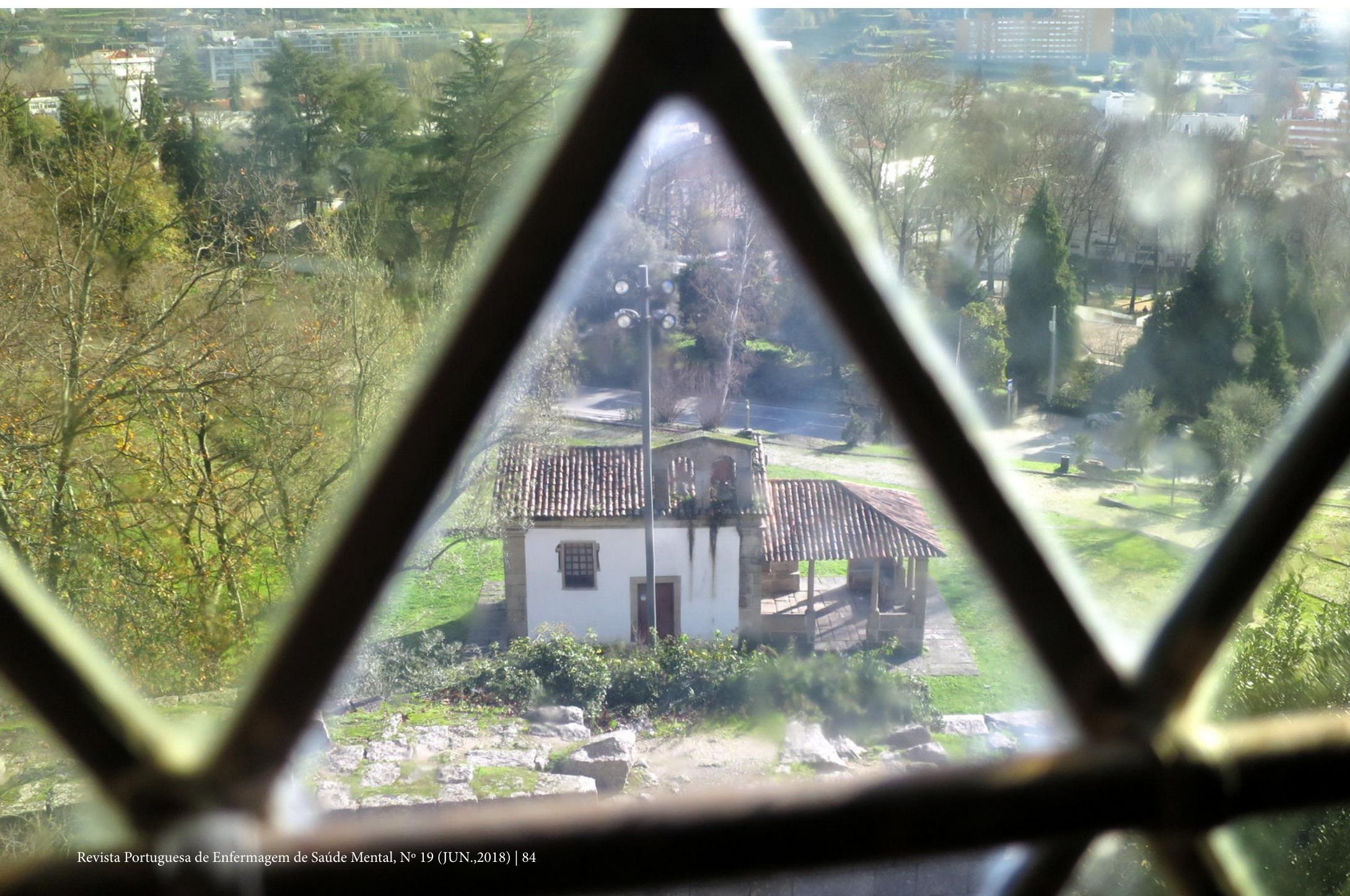

